Tibor Kiss

\title{
Bedingungen für den Wegfall eines Artikels: Distribution und Interpretation von Präposition-Nomen-Kombinationen ${ }^{1}$
}

\begin{abstract}
Kombinationen aus Präposition und artikelloser Nominalprojektion, deren syntaktischer Kopf ein zählbares Substantiv im Singular ist, fristeten lange Zeit ein Schattendasein in der Grammatikschreibung. Sie wurden ignoriert oder als Ausnahmen beschrieben, obwohl sie offenkundig regelhaft gebildet werden. Im vorliegenden Aufsatz verwenden wir computerlinguistische Verfahren, insbesondere „Annotation Mining" und logistische Regression, um die syntaktische Distribution dieser Kombinationen zu charakterisieren und anhand zweier Präpositionen (obne und unter) detailliert die Realisationsbedingungen zu bestimmen.
\end{abstract}

\section{Einleitung}

Präpositionen werden in vielen Sprachen mit Nominalphrasen kombiniert. Viele Präpositionen gestatten jedoch auch eine Kombination mit einem artikellosen Substantiv oder einer artikellosen Substantivgruppe, wie die Beispiele in (1) zeigen.

(1) auf parlamentarische Anfrage, bei absolut klarer Zielsetzung, in untertreibender Anspielung, mit schwer beladenem Rucksack, ohne mündliche Vorwarnung, unter sanfter Androhung

Die Beispiele in (1) sind bemerkenswert, denn die Substantive werden als qüblbar analysiert und dürften infolge dieser Eigenschaft eigentlich nicht ohne Artikel realisiert werden. So stellt der Duden (2005, S. 337) in der Regel 442 fest, dass „Substantive mit Merkmalkombination ,zählbar ${ }^{6}$ plus Singular ... grundsätzlich immer ein Artikelwort bei sich [haben], und wenn es als letzte Möglichkeit der indefinite Artikel ist."

\footnotetext{
Die vorliegenden Ergebnisse entstammen dem Projekt „Erfassung und Analyse syntaktischer und semantischer Eigenschaften von Präposition-Substantiv-Sequenzen unter Verwendung computerlinguistischer Analyseverfahren", das seit 2009 freundlicherweise von der DFG gefördert wird. Die Ergebnisse hätten ohne die Mitarbeit von Antje Müller, Claudia Roch, Tobias Stadtfeld, Katja Keßelmeier und Jan Strunk nicht erzielt werden können. Ich danke ihnen herzlich. Durch die Einladung zur IDS-Jahrestagung 2010 hatte ich die Gelegenheit, Teile des vorliegenden Aufsatzes zu präsentieren. Ich danke Stefan Engelberg und den Organisatoren für die Einladung, Anke Holler, Kristel Proost und Stefan Engelberg für ihre Kommentare und den Zuhörern für die Diskussionsbeiträge.
} 
Deutlich wird anhand der Beispiele in (1) auch, dass es sich hierbei nicht um Paarbildungen aus Präposition und Substantiv handelt: Pränominale Modifikation ist in vielen Fällen möglich, teilweise auch durchaus komplex. Dieser Eindruck wird noch dadurch verstärkt, dass postnominale Komplemente nicht ausgeschlossen sind:

(2) Riess-Passer unterstrich die Forderung nach Stillegung des grenznahen Atomkraftwerks Temelin in Tschechien. (NZZ, AUSLAND, 30.11.2001)

Das Beispiel in (2) zeigt darüber hinaus, dass die fraglichen Konstruktionen nicht nur adverbial verwendet werden können, sondern auch in regierten Kontexten erscheinen - die Präposition nach wird in (2) durch Forderung regiert.

In der Folge bezeichne ich Kombinationen wie die in (1) und (2) als Präposition-Nomen-Kombinationen (PNKen). PNKen wurden in der Grammatikschreibung lange ignoriert oder als Ausnahmen charakterisiert; in den letzten Jahren hat sich die Position aber sowohl aus typologischer als auch aus grammatiktheoretischer Perspektive gewandelt. Himmelmann (1998, S. 316) hat beobachtet, dass PNKen in vielen Sprachen auftreten, deren Grammatiken verlangen, dass zählbare Substantive im Singular durch einen Artikel ergänzt werden müssen. Stvan (1998) bietet für das Englische eine Analyse an, die auf den semantischen Eigenschaften des Substantivs basiert; Baldwin et al. (2006) entwickeln eine Typologie der Konstruktion für das Englische und stellen beiläufig fest, dass es sich zumindest in Teilen um eine produktive - im syntaktischen Sinne also regelhafte - Konstruktion handelt, die durch Ausnahmen nicht erfasst werden kann. ${ }^{2}$ Während der Duden (2005) die Konstruktion als Ausnahme beschreibt, zeigen Kiss (2007) und Dömges et al. (2007) anhand eines quantitativen Produktivitätsmaßes, dass es sich um eine produktive und somit regelhafte Kombination handelt, die somit auch durch die Grammatik erfasst werden muss.

PNKen stellen so die Grammatikschreibung vor eine Herausforderung: Es besteht einerseits weitgehender Konsens darüber, dass die Kombination in vielen Sprachen regelhaft ist; andererseits ist aber auch klar, dass die zugrunde liegenden Regeln offensichtlich nicht dem Typ entsprechen, der etwa die Kombination einer DP mit einem transitiven Verb steuert. ${ }^{3}$ Introspektive Urteile zur Kombinierbarkeit von Präposition und artikelloser

Studien zu PNKen im Englischen bezeichnen die Konstruktion als „,determinerless PPs“ (Baldwin et al. 2006) oder „bare PPs“ (Le Bruyn et al. 2009).

3 Ich gehe im Folgenden davon aus, dass vollständige Nominalprojektionen, die einen Artikel enthalten, DPen sind. Die Festlegung ist in vorliegenden Kontext schon deswegen nützlich, weil so Nominalprojektionen mit Artikel notationell von Nominalp rojektionen ohne Artikel unterschieden werden können. Eine Festlegung auf eine bestimmte Analyse der Nominalprojektion ist damit nicht verbunden. 
Nominalgruppe werden nur zögerlich getroffen. Sprecher sind ebenfalls sehr zögerlich, neue PNKen zu bilden. Dem steht wiederum die quantitative Produktivität des Prozesses gegenüber - eigentlich ein Widerspruch!

Zur Identifikation der Grammatikalitätsbedingungen der PNKen wähle ich das „Annotation Mining" (vgl. Chiarcos et al. 2008). Es bietet sich als exploratives Analyseverfahren an, in dem auf die Identifikation oder Elizitation von Grammatikalitätsurteilen verzichtet wird. Grammatikalitätsbedingungen werden aus umfangreichen Sprachdaten abgeleitet, die wiederum durch Merkmale auf den unterschiedlichen linguistischen Ebenen annotiert sind (Lexikon, Syntax, Semantik, globaler Kontext). Die annotierten Daten werden Klassifikationsverfahren aus der inferentiellen Statistik unterworfen, im vorliegenden Fall insbesondere der binären logistischen Regression (Harrell 2001), die in Abschnitt 4.2 vorgestellt wird.

Anhand von zwei Pilotstudien zur Syntax der Präpositionen ohne und unter möchte ich erläutern, warum die Ansätze von Stvan (1998) und Baldwin et al. (2006) grundsätzlich in die richtige Richtung weisen, aber dann eben doch nicht auf das Deutsche übertragbar sind. Die Modelle zeigen, dass es unabhängig von der Interpretation der Präpositionen strukturelle Faktoren gibt, die die Weglassbarkeit des Artikels zumindest partiell bedingen.

\section{Zur Grammatik von Präposition-Nomen-Kombinationen}

Die hier verwendete Definition von PNKen ist gegenüber anderen Definitionen durch eine interne und eine externe Bedingung eingeschränkt. Obwohl diese Einschränkungen eigentlich zwingend mitgedacht werden müssen, zeigen etwa die Untersuchungen von Le Bruyn et al. (2009) - in denen weder die interne noch die externe Bedingung eine Rolle spielt - dass es erforderlich ist, die Bedingungen explizit zu machen. Die interne Bedingung sieht vor, dass unter PNKen nur solche Kombinationen aus einer Präposition und einer artikellosen Nominalprojektion fallen, in denen das Substantiv ein zäblbares Substantiv im Singular ist. Weder PPen mit Massentermen noch PPen mit nackten Pluralia fallen so unter die PNKen. Da im Deutschen Artikel bei Massentermen nicht obligatorisch sind - Indefinita sind sogar untersagt - und bei Pluralia zumindest optional, entstehen durch den Wegfall des Artikels in diesen Phrasen keine Probleme. Betrachtete man die Distribution des Artikels bei Pluralia und Massentermen in anderen Sprachen, etwa den romanischen Sprachen (vgl. Espinal/McNally ersch. 2011), so würden andere Definitionen erforderlich.

Für das Deutsche liegt zunächst aber die Tücke im Detail: Zum einen setzt die Definition ein Konzept der Zählbarkeit voraus, zum anderen handelt es sich hierbei bestenfalls um eine notwendige Definition, nicht aber um eine hinreichende, denn es kann nicht jede Präposition mit jedem Substantiv in einer PNK kombiniert werden. 
Die externe Bedingung setzt voraus, dass es sich bei der Präposition in der PNK um eine Präposition handelt, die überhaupt DP-Objekte besitzen kann. Auch diese Annahme ist nicht trivial. Sie führt dazu, dass wir nicht alle Präpositionen des Deutschen untersuchen, sondern nur einen Teilbereich der Präpositionen, nämlich solche, die DP-Objekte besitzen und in PNKen auftreten können. Diese Präpositionen sind in (3) aufgeführt; in Abschnitt 2.3 werde ich anhand der Präposition per das Verhalten von Kombinationen aus Präposition und Substantiv erläutern, die dieser Bedingung nicht genügen. Die in (3) aufgeführten Präpositionen erfüllen das externe Kriterium, allerdings nicht mit jedem zählbaren Substantiv im Singular, wie anhand von (4) verdeutlicht werden kann. ${ }^{4}$

(3) an, auf, bei, dank, durch, für, gegen, gemäß, hinter, in, mit, mittels, nach, neben, ohne, seit, über, um, unter, vor, während, wegen

(4) *Riess-Passer wehrt sich gegen Forderung nach Stilllegung des grenznahen Atomkraftwerks Temelin in Tschechien.

Das Beispiel in (4) ist eine Variation des Beispiels in (2), bei der auch auf den wohl tatsächlich obligatorischen Artikel vor Forderung verzichtet wurde. Auch semantisch sehr nahe Substantive können in PNKen nicht einfach ausgetauscht werden, wie anhand der Substantive Voraussetzang und Prämisse in (5) und (6) gezeigt werden kann.

(5) Auch Philipp Egli besteht auf einer eigenen Handschrift - unter der Voraussetzung/der Prämisse des Einverständnisses des Ensembles.

(6) Auch Philipp Egli besteht auf einer eigenen Handschrift - unter Voraussetzung/*Prämisse des Einverständnisses des Ensembles.

Während Voraussetqung mit und ohne Artikel als Komplement von unter erscheinen kann, ist bei Prämisse nur die Realisation in einer PP möglich.

Der Kontrast zwischen (5) und (6) könnte durch zwei Muster erklärt werden: entweder durch einen Rekurs auf eine entsprechende Definition der Zählbarkeit, oder durch den Nachweis tatsächlich fehlender Regelhaftigkeit der Konstruktion. Diese Punkte wollen wir in den folgenden Abschnitten diskutieren.

\subsection{Das Problem der Zählbarkeit}

Die Dudenregel 442 (Duden 2005, S. 337) besagt, dass zählbare Substantive im Singular im Deutschen durch einen Artikel begleitet werden müssen. Daraus folgt nicht nur, dass die Beispiele in (1) und (2) ungrammatisch sein

Das Beispiel (4) wird dann nicht als ungrammatisch empfunden, wenn es als Überschrift eines Zeitung-sartikels verwendet würde. In Überschriften findet man häufig PNKen, die außerhalb von Überschriften als deutlich abweichend bewertet werden. 
sollten. Die Regel setzt auch voraus, dass die Eigenschaft der Zählbarkeit definiert wird. Zählbarkeit könnte als lexikalische Abbildung von Worten auf Denotate verstanden werden. Sind die Denotate zählbar, dann ist auch das Wort zählbar. Diese Definition geht zumindest auf Jespersen (1924) zurück und fand auch in der generativen Grammatik Rückhalt. Sie führt aber zu einer Vielzahl von Problemen. Hier ist nicht der Raum, um dies auch nur andeutungsweise zu erläutern, es soll nur erwähnt werden, dass es nach dieser Definition keine Synonyme $S_{1}$ und $S_{2}$ in einer Sprache geben sollte, bei denen $S_{1}$ zählbar ist, $S_{2}$ aber nicht. ${ }^{5}$ Darüber hinaus kommt diese Definition in Schwierigkeiten, wenn ein zählbares Wort nicht zählbar verwendet wird, man vergleiche hierzu die Verwendung von Fabrzeug vs. Fahrzeuge in (7).

(7) Hier bekommen Sie mehr Fahrzeug/Fahrzeuge für ihr Geld.

Allan (1980) hat eine alternative Sichtweise des Konzepts der Zählbarkeit in der Sprachwissenschaft etabliert, die in jüngeren Arbeiten von Borer (2005) und Bale und Barner (2009) auch formal expliziert wurde. Nach dieser Auffassung ist Zählbarkeit keine lexikalische Eigenschaft, sondern eine Eigenschaft nominaler Phrasen, die durch kontextuelle formale Merkmale zugewiesen wird, etwa die Pluralmorphologie oder die Realisation eines Artikels.

Dieser Idee folgend ist ein Nomen zunächst einmal weder zählbar noch nicht zählbar. Wird dieses Nomen in einen entsprechenden Kontext eingesetzt, ist die resultierende Phrase aber zählbar oder nicht. Für das Beispiel (7) bedeutet dies, dass Fahrzeng, wenn es weder flektiert noch durch einen Artikel begleitet wird, als Massenterm analysiert wird. Das Pluralmorphem bildet hingegen einen Kontext für Zählbarkeit, ebenso würde ein solcher Kontext durch einen singularischen oder pluralischen Artikel geschaffen. Für die Syntax der PNKen ergibt sich nun allerdings eine merkwürdige Konsequenz: Da hier die relevanten Substantive in Kontexten realisiert werden, in denen weder ein Artikel noch ein Pluralmorphem vorliegt, wird die Dudenregel 442 schon deswegen nicht verletzt, weil ein Substantiv in einer PNK nach dieser Definition nicht zählbar ist. Dies bedeutet aber auch, dass jedes Substantiv in einer PNK realisiert werden können müsste, weil hier nie ein Kontext für Zählbarkeit vorliegt. Konsequenterweise sollten sich PNKen also wie PPen verhalten und ihre besondere Betrachtung wäre überflüssig. Dass die Schlussfolgerung nun wieder voreilig ist, folgt aus zwei Beobach-

Der Leser erwartet hier sicherlich eine Stellungnahme, aus der hervorgeht, ob es nun entsprechende Synonyme gibt oder nicht. Anhand von Beispielen wie Fußbekleidung vs. Schube könnte man diese Frage nun positiv beantworten. Allerdings ist zu berücksichtigen, dass das Konzept der Synonymie nicht präzise definiert ist. Es wird jedoch auch niemand bestreiten wollen, dass es sehr schwer werden würde, Modelle zu konstruieren, in denen die Aussagen (i) und (ii) unterschiedliche Wahrheitswerte besitzen:

(i) Du hast aber viele Schuhe.

(ii) Du hast aber viel Fußbekleidung: 
tungen: Zum einen haben wir bereits gesehen, dass nicht jedes Substantiv in einer PNK realisiert werden kann; zum anderen würde dies auch bedeuten, dass jedes Substantiv in einer PNK die Interpretation eines Massenterms erhält. Dies ist aber ebenfalls falsch, wie man an Beispielen wie (8) und (9) sieht.

(8) Milosevic unterschrieb auch unter $\oslash /$ der/einer Androhung von NATO-Bombardementen nicht.

In (8) können wir beobachten, dass bei den Realisationen als PP (DP mit definitem oder indefinitem Artikel) oder PNK die gleiche Interpretation hervorgerufen wird. Es ist dann die plausiblere Überlegung, dass bei einer Realisation mit Artikel tatsächlich Zählbarkeit vorliegt und somit Bedeutungsgleichheit auch bei einer Realisation ohne Artikel. Die gleiche Argumentation gilt auch für die mit durch eingeleitete Phrase in (9).

(9) Ursprünglich war der Artikel als Verbot der Beleidigung jeder Religion eingeführt worden, der Diktator engte ihn jedoch nach 1980 auf die Beleidigung des Islam ein, und er verschärfte ihn durch $\varnothing /$ eine/ die Androhung der Todesstrafe. (NZZ, AUSLAND, 25.02.1995, Originalbeleg mit definitem Artikel)

Es zeigt sich hier, dass die kontextuelle Definition der Zählbarkeit ebenso problematisch ist wie die lexikalistische Position. Darüber hinaus ist es notwendig zu erläutern, was im Rahmen einer automatischen Klassifikation eigentlich unter einem zäblbaren Substantiv zu verstehen ist, wenn weder gänzlich auf den Typ noch gänzlich auf die einzelnen Vorkommen rekurriert werden kann.

Die Zählbarkeitsklassifikation basiert auf zwei statistischen Klassifikatoren, die einerseits das Verhältnis von Plural und Singularvorkommen betrachten und andererseits syntaktische Kontexte (die durch die POS-Tags vor dem zu klassifizierenden Nomen identifiziert werden, vgl. Abschnitt 3). Diese Klassenbildung erfolgt nach einem Vorschlag von Stadtfeld (i.Vorb.), basierend auf den Arbeiten von Gillon (1999) und Barner/Snedeker (2005). Stadtfeld unterscheidet fünf Zählbarkeitsklassen (I = zählbar, II = Pluralia tantum, III = echt ambig, IV und V = nicht zählbar), die nach drei Kriterien identifiziert werden können.

Das erste Kriterium - der Messmodus - erfasst, ob ein Singularvorkommen eines Nomens mit mehr hinsichtlich einer definierten Quantität verglichen werden kann. Für echt zählbare Substantive (Klasse I) ergibt dies keinen Sinn, für nicht-zählbare Substantive der Klasse IV ist ein Vergleich hinsichtlich Quantität von Individuen oder einer anderen Messgröße möglich, für nicht-zählbare Substantive der Klasse V ist ein Vergleich nur hinsichtlich einer nicht auf Individuen quantifizierenden Messgröße möglich. 


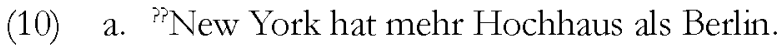

b. Paul hat mehr Besteck als Peter.

c. Paul hat mehr Reis als Peter.

Das Beispiel (10a) ist auch dann inakzeptabel, wenn vorher bekannt ist, dass das Gesamtgewicht der Hochhäuser in New York höher ist als das Gewicht aller Hochhäuser Berlins (Beispiele dieses Typs findet man eigentlich nur in Werbekontexten). Das Beispiel (10b) kann wahr sein, wenn es tatsächlich insgesamt mehr individuelle Besteckelemente gibt oder wenn Paul mehr Bestecktypen als Peter besitzt. Das Beispiel (10c) setzt voraus, dass die MessgröBe das Gesamtvolumen ist. Individuen sind also bei Klasse V ausgeschlossen, führen aber bei Klasse IV zu einer Typ- bzw. Behälterinterpretation.

Der zweite Test überprüft verborgene Typlesarten bei Pluralrealisationen. Auch hier erfolgt eine Einbettung des Nomens unter mehr, das Nomen wird nun aber im Plural realisiert. Bei Massentermen der Klasse V ist der Test nicht anwendbar, weil sie keine Pluralrealisation besitzen, bei Massentermen der Klasse IV ergibt die explizite Realisation einer Numeralkonstruktion eine äquivalente Lesart (11), bei zählbaren Substantiven der Klasse I ist diese Äquivalenz nicht gegeben (12).

(11) a. Paul hat mehr Weine als Peter.

b. Paul hat mehr Sorten Wein als Peter.

(12) a. Ulrich vertreibt mehr Weingläser als Paul.

b. Ulrich vertreibt mehr Sorten Weinglas als Paul.

Der letzte Test betrifft die Verwendung des indefiniten Artikels in Kontexten der Form indefiniter Artikel $+N$ ist ein Hyperonym. Bei zählbaren Substantiven der Klasse I ist der indefinite Artikel obligatorisch (13), die Klassen IV und V gestatten die Realisation des indefiniten Artikels nicht (14), es kommt zu Typlesarten, die im vorgegebenen Kontext blockiert sind, weil hier allgemeine Eigenschaften das Prädikat bilden.

(13) a. Ein Auto ist ein Fahrzeug.

b. *Auto ist ein Fahrzeug.

(14) a. Stahl ist eine Metalllegierung.

b. ${ }^{? P}$ Ein Stahl ist eine Metallegierung.

(15) a. Besteck ist ein Werkzeug.

b. ${ }^{? ?}$ Ein Besteck ist ein Werkzeug.

Ein wesentlicher Vorteil dieses feineren Klassifikationsansatzes ist die Identifikation echt mehrdeutiger Substantive, die in der bislang nicht diskutierten Klasse III erfasst werden. Diese Substantive sind für eine binäre Klassifikation insofern problematisch, als sie Eigenschaften von qüblbaren und nicht- 
rählbaren Nomina aufweisen. So gestatten Substantive wie Kuchen oder Fisch die Verwendung des indefiniten Artikels (16a), was für die Zählbarkeit spricht, zugleich kann aber der Artikel auch weggelassen werden, wie (16b) zeigt.

(16) a. Ein Kuchen ist ein Grundnahrungsmittel.

b. Kuchen ist ein Grundnahrungsmittel, meinte schon MarieAntoinette.

PNKen, in denen das Substantiv der Klasse III angehört, werden zunächst aus der Analyse ausgeschlossen, bis die Charakteristika dieser Klasse genauer bestimmt sind. Erfreulicherweise enthält diese Klasse wenige Elemente.

In der folgenden Analyse (vgl. Abschnitte 3 und 4) bezeichne ich die in den PNKen auftretenden Substantive als zählbar, weil sie der Klasse I und als nicht-zählbar, wenn sie den Klassen IV oder V zugeordnet werden konnten. Eine binäre Opposition wird damit nicht behauptet.

\subsection{Produktivität}

Wir können somit davon ausgehen, dass zählbare Substantive in PNKen realisiert werden, ein Verweis auf eine mögliche Analyse als Massenterme ist falsch und hilft nicht weiter. Aus dieser Annahme würde folgen, dass Nomina in PNKen grundsätzlich als Massenterme interpretiert würden - und somit PNKen auch grundsätzlich grammatisch sein sollten, wobei eine Unakzeptabilität dann entstünde, wenn eine Massenterminterpretation inkompatibel wäre.

Ein weiterer Ausweg könnte natürlich darin bestehen, die Konstruktionen insgesamt als Ausnahmen zu analysieren. Dies ist etwa der Stand der Dinge im Duden: Die Regel 395 zählt die Ausnahmen auf, die die Realisation eines zählbaren Substantivs ohne Artikel gestatten. Kurz gesagt: Die hier bislang vorgestellten, zweifelsfrei grammatischen Beispiele zählen nicht dazu.

Diese Feststellung soll aber nicht genügen. Basierend auf dem morphologischen Produktivitätsmaß P aus Baayen (2001) und dessen Verallgemeinerung für syntaktische Kontexte in Evert (2004) legen Dömges et al. (2007) eine quantitative Analyse der Produktivität von PNKen mit unter vor, aus der hervorgeht, dass es sich bei PNKen um regelhafte Kombinationen handelt. Ohne die technischen Details dieser Analyse hier zu diskutieren, kann der Ansatz wie folgt zusammengefasst werden: Wenn man davon ausgehen würde, dass eine syntaktische Kombination tatsächlich eine aufzählbare Ausnahme ist, dann müsste es auch endlich viele Instanzen dieser Ausnahme geben. Dies wiederum würde bedeuten, dass bei einem genügend großen Korpus irgendwann einmal beobachtet werden könnte, dass das Vokabular für diese spezielle Konstruktion nicht mehr anwächst (während 
bei regelhaften, nicht aufzählbaren Konstruktionen ein stetiges Vokabularwachstum beobachtbar ist). Wenn wir nun eine Präposition als Beispiel auswählen, dann besteht das fragliche Vokabular aus Substantiven, die mit dieser Präposition kombiniert werden können. In diesem Sinne wächst das Vokabular von unter Androhung auf unter sanfter Androhung nicht an, weil wir hier das Substantiv Androhung bereits gesehen haben. Anders ist dies, wenn wir zunächst unter Androhung und dann unter Auswertung oder auch unter Gewaltandrohung beobachten würden.

Konkretisiert auf eine Präposition wie unter bedeutet dies, dass zu irgendeinem Zeitpunkt $\mathrm{t}_{1}$ die Wahrscheinlichkeit, dass zu Folgezeitpunkten $\mathrm{t}_{1+\mathrm{n}}$ noch neue Instanzen von unter + Substantiv beobachtet werden können, unter einen kritischen Wert fällt, wenn der Prozess nicht produktiv ist.

Diese Annahme kann nun von beobachteten Daten auf nicht beobachtete Daten, also von bereits sehr umfangreichen empirischen Korpora auf wesentlich umfangreichere nicht beobachtete Korpora übertragen werden, wenn man so genannte LNRE-Modelle (, large number of rare events", Baayen 2001) zur Vorhersage nicht beobachteter Daten verwendet. Dömges et al. (2007) haben gezeigt, dass das plausibelste Modell für unter+Substantiv dasjenige Modell ist, das unendlich viele Instanzen dieser Kombination vorhersagt. Somit ist die Kombination regelhaft, auch der Weg über die Ausnahme ist verbaut.

\subsection{Eine Analyse des Englischen, die im Deutschen nicht funktioniert}

Für PNKen im Englischen schlägt Stvan (1998) vor, dass es einen Prozess der N-Selektion gibt. Nach dieser Analyse werden PNKen durch die in ihnen enthaltenen Substantive lizenziert, wie anhand der Substantive school, jail und prison in (17) illustriert werden kann.

$$
\text { from school, at school, in jail, from jail, in prison, from prison ... }
$$

Baldwin et al. (2006) legen Stvans Analyse zugrunde und argumentieren, dass es sich bei den hier realisierten Substantiven häufig um solche handelt, die auch in anderen syntaktischen Kontexten ohne Artikel realisiert werden können, obwohl die Artikelrealisation eigentlich obligatorisch ist. Als Beispiel mag hier das Substantiv school in (18) dienen, das als Subjekt eines kategorischen Satzes artikellos realisiert wird. Baldwin et al. (2006) bezeichnen solche Substantive als ,defective nouns" und charakterisieren sie semantisch als ,institutional nouns".

(18) School is over.

Stvan (1998) beobachtet nun weiterhin, dass bei einer Realisation eines Substantivs in einer PNK eine pragmatische Bedeutungserweiterung erfolgt. So bedeutet das Beispiel in (19a) nicht allein, dass Marys Gatte im Gefängnis 
verortet ist, sondern vor allem, dass Marys Gatte in diesem Gefängnis eine Haftstrafe verbüßt; (19b) bedeutet nicht nur, dass John in einer Schule lokalisiert werden kann, sondern, dass eine institutionell geprägte kontextuell näher zu explizierende Beziehung zwischen John und der Schule besteht.

a. Mary's husband is in prison.

b. John is at school.

Baldwin et al. (2006) schlagen neben PNKen mit N-Selektion auch PNKen mit P-Selektion vor. Sie charakterisieren solche PNKen durch semantische Selektionsregeln die den Typ des nominalen Komplements einschränken, wobei sie implizit davon ausgehen, dass durch die semantische Selektion auf das Substantiv auch die Polysemie der Präposition reduziert wird (zur Polysemie siehe Abschnitt 3). Diese Selektion ergibt dann produktive Muster wie die in (20) und (21).

(20) by train, by plane, by bus, by pogo stick, by hydro-foil, ...

(21) on disc, on CD, on DVD, on tape, on stick, on memory card, ...

Es stellt sich dann natürlich die Frage, ob die Vorschläge von Stvan (1998) und Baldwin et al. (2006) vom Englischen auf das Deutsche übertragen werden können.

Zunächst ist hier festzuhalten, dass es produktive Muster des Typs (20) und (21) auch im Deutschen gibt, aber gerade nicht bei PNKen, so wie ich sie definiert habe. Eine Übersetzung von by ins Deutsche würde wahrscheinlich die Präposition per ergeben, die dann in der Tat Muster wie in (22) zeigt.

(22) per Zug, per Flugzeug, per Bus, per Skateboard, ...

Hier greift nun die externe Bedingung der Definition der PNKen: Die Präposition per besitzt im Deutschen überhaupt keine DP-Objekte, d.h. Objekte mit realisiertem Artikel. Somit handelt es sich bei den Beispielen in (15) nicht um PNKen. Muster des Typs (20) werden im Deutschen durch DPen mit definitem Artikel realisiert, so wie man in (23a) sehen kann. Diese Kombination ist auch ohne Präposition verwendbar, wie (23b) zeigt, aber niemals ohne Artikel (24). ${ }^{6}$

\footnotetext{
Hier mag man einwenden, dass Fabren wir doch mit Bus und Bahn grammatisch ist und somit der oben genannten Behauptung widerspricht. Der Grammatikalität dieses Beispiels liegt allerdings eine andere Regularität zugrunde: Mit oder oder und verknüpfte artikellose Aufzählungen von Substantiven sind nach Präpositionen des Deutschen grundsätzlich möglich. Obwohl dieser Teilbereich der Grammatik der Präpositionen noch nicht gut untersucht ist, so kann man doch festhalten, dass Aufzählungen zu einer Pluraldenotation führen und somit die Beschränkungen für artikellose Pluralia im Deutschen gelten. Ich denke, dass ein Beispiel wie etwa Ich fuhr mit Bus und Babn nicht wahr sein kann, wenn ich tatsächlich nur mit dem Bus (oder nur mit der Bahn) gefahren bin.
} 
(23) a. Fahren wir mit dem Bus/mit dem Zug/mit dem Skateboard?

b. Nehmen wir den Bus/den Zug/das Skateboard?

a. *Fahren wir mit Bus/mit Zug/mit Skateboard?

b. *Nehmen wir Bus/Zug/Skateboard?

In den Beispielen in (23) wird keineswegs vorausgesetzt, dass ein Fahrzeug salient, einzigartig oder vorerwähnt wäre.

Die von Stvan (1998) beobachtete pragmatische Bedeutungserweiterung findet sich im Deutschen nicht oder kaum in PNKen, wohl aber in Verschmelzungsformen wie (25).

(25) Er ist im Gefängnis.

Dass PNKen nicht notwendigerweise mit Bedeutungsveränderungen korrespondieren, hatten wir bereits anhand der Beispiele in (8) und (9) verdeutlicht, auf die hier nochmals verwiesen werden soll. Wenn hier eine artikellose Phrase eine Bedeutung besitzt, die nahezu identisch zu einer Phrase mit Artikel ist, dann kann eine pragmatische Bedeutungserweiterung kein konstitutiver Bestandteil von PNKen im Deutschen sein.

Auch nicht beobachten lassen sich ,defekte Nomina“. Beispiele des Typs (18) verlangen im Deutschen einen definiten Artikel. Bereits in Iinsicht auf die so genannten, ,institutional nouns" (school, church) stellen Baldwin et al. (2006, S. 170) unter Verweis auf Himmelmann (1998) fest, dass ,,the fact that institutional nouns can occur without determiners in these environments is, however, a peculiarity of English; related Germanic languages such as German or Swedish require the definite article here."

Die Schule ist aus.

Während wir für die hier diskutierten Beispiele wohl zurecht behaupten können, dass eine einfache Übertragung der Ansätze von Stvan (1998) und Baldwin et al. (2006) auf das Deutsche nicht möglich ist, sollte die generelle Idee, dass die Semantik der beteiligten Elemente eine konstitutive Rolle spielt, weiter überprüft werden. Tatsächlich haben wir in unserer Analyse die Einbeziehung der Semantik der Präposition und der Semantik des Substantivs vorgesehen und es wird sich zeigen, dass beide eine Rolle spielen.

Wir schließen aber zunächst unsere Betrachtung mit der Schlussfolgerung, dass Analysen dem Phänomenbereich nicht gerecht werden, die entweder das Substantiv als nicht zählbar oder PNKen insgesamt als Ausnahmen klassifizieren. Ebenso wenig ist eine einfache Übertragung der Analysen aus dem Englischen auf das Deutsche möglich. Und schließlich muss auch nochmals betont werden, dass sich die Konstruktionen der reinen Introspektion entziehen. Eine Alternative bietet hier das Annotation Mining. 


\section{Annotation Mining}

Die vorliegende Analyse basiert auf einem Zeitungskorpus, den Jahrgängen von 1993 bis 1999 der Neuen Zürcher Zeitung mit einem Umfang von ca. 230 Millionen Wörtern. Die gesamte Annotation basiert auf einem XMLStand-Off-Format, Daten und Annotate sind also strikt voneinander getrennt, was sowohl die Extraktion einzelner Annotationsebenen als auch ihr Hinzufügen deutlich vereinfacht. Als Werkzeug für die manuelle Annotation insbesondere der Präpositionsbedeutungen verwenden wir MMAX2 (Müller/Strube 2006).

$\mathrm{Da}$ es das Ziel der vorliegenden Analyse ist, konstitutive Bedingungen für die Realisation von PNKen insbesondere auch in Abgrenzung zur Realisation in der Form einer PP zu identifizieren, werden nicht nur PNKen annotiert, sondern auch PPen, für die gilt, dass Präposition und Substantiv in einer PNK aufgetreten sind, und PPen, die ein zählbares Substantiv einbetten, das nicht in einer PNK mit derselben Präposition aufgetreten ist.

Für diese Daten sehen wir die folgenden Annotationen vor:

- Lexikalische Ebene: Part-of-Speech-Tags (kategoriale Etikettierung), Flexionsmorphologie, Derivationsmorphologie von Substantiven, Zählbarkeit von Substantiven, Interpretation von Substantiven durch GermaNet-Tops (d.h. Wortfelder), Interpretation von Präpositionen, Kompositabildung bei Substantiven.

- Syntaktische Ebene: Einbettungsmodus (Adjunkt oder Komplement) der PP/PNK, syntaktische Komplemente des Nomens, pränominale Modifikatoren des Nomens.

- Globale Ebene: Ist die Phrase Bestandteil einer Überschrift, eines Titels oder eines Zitats? Ist die Phrase idiomatisch?

In Überschriften, Titeln und Zitaten könnten PNKen nicht aufgrund syntaktischer oder semantischer Bedingungen realisiert werden, sondern weil Überschriften und Titel typische Orte für extragrammatische Kürzungen sind. Rein idiomatische PNKen mögen Kombinationsregeln folgen, die von den generellen Bedingungen für die Realisation von PNKen abweichen. Die Annotation dient auf der globalen Ebene also wesentlich dazu, bei der Analyse einzelne Fälle auszuschließen.

Große Teile der Annotation erfolgen automatisch mit der Hilfe der folgenden Werkzeuge: Der „Regression Forest Tagger“ (Schmid/Laws 2008) wird verwendet für die kategoriale Etikettierung und die flexionsmorphologische Analyse (der Tagger enthält die morphologische Analysekomponente SMOR, vgl. Schmid 2004), der „Tree Tagger" (Schmid 1995) wird für die flache syntaktische Verarbeitung („Chunk Parsing") verwendet. Derivationsmorphologische Kategorien werden aus IMSLex (Lezius et al. 2000) abgeleitet. 
Die Analyse verwendet zwei Ressourcen, um die Interpretation der Substantive festzulegen. Die erste Ressource ist GermaNet (Kunze/Lemnitzer 2002), die deutsche Version des WordNet (vgl. http://wordnet.princeton.edu/). Wir verwenden die 23 Top-Level-Kategorien und jedes Substantiv wird mit allen Kategorien annotiert, denen es angehört. Top-Level-Kategorien entsprechen Wortfeldern. Darüber hinaus verwenden wir das Lexikon HaGenLex (Hartrumpf et al. 2003). HaGenLex kodiert spezifische sortale Informationen, die in einer formalen Ontologie strukturiert sind.

Die Klassifikation der Zählbarkeit erfolgt so, wie in Abschnitt 2.1 bereits beschrieben wurde. Zählbare Substantive sind solche, die der Klasse I angehören, nicht-zählbare Substantive sind solche, die den Klassen IV bzw. $\mathrm{V}$ angehören.

Durch automatische Annotationsverfahren liegen somit Informationen vor, die bei den Nomina von der internen Struktur über die syntaktische Distribution bis hin zur sortalen Klassifikation reichen. Leider liegt ein entsprechendes Verfahren für die Interpretation der Präpositionen nicht vor. Obwohl der Grad der Polysemie der Präpositionen nahezu ein Gemeinplatz ist, sind bislang keine Versuche unternommen worden, Kategorieninventare (,Sense Tags') für Präpositionsinterpretationen zu entwickeln. Sowohl die manuelle als auch die automatische Annotation setzen aber ein vordefiniertes Kategorieninventar voraus. Dieses Inventar muss natürlich die Polysemie der Präpositionen abbilden, darf aber nicht so komplex sein, dass die manuelle Annotation unmöglich wird. So legt etwa Schröder (1986) eine umfangreiche merkmalsbasierte Bestimmung der Interpretation von Präpositionen vor, die aber auf ca. 200 Merkmalen basiert und daher nicht für die Annotation eingesetzt werden kann. Dennoch ist Schröders Lexikon eine wertvolle Ressource.

Unsere Analyse basiert neben Schröder (1986) auf drei weiteren, im Wesentlichen gebrauchsorientierten Ressourcen zur Beschreibung der Präpositionsinterpretationen des Deutschen: der Analyse von Helbig und Buscha (2001), der Darstellung im Duden „Deutsch als Fremdsprache“ (2002) und der Arbeit von Durrell und Brée (1993) zur Interpretation temporaler Präpositionen.

$\mathrm{Zu}$ diesen Ressourcen ist zu sagen, dass sie sehr heterogen und teilweise eher beispielorientiert als regelbasiert sind. Vor einer Anwendung ist also zunächst eine Angleichung und kritische Überprüfung der genannten Inventare erforderlich. Dies führt häufig zu einer Erweiterung von Interpretationsmerkmalen für einzelne Präpositionen und nicht selten zu einer Neudefinition von Teilen des Inventars. Das Annotationsschema für Präpositionsinterpretationen wird in Müller et al. (i.Dr.) detailliert beschrieben; ich möchte es an dieser Stelle anhand der wesentlichen Merkmale charakterisieren: 
- Es gibt ein Inventar von insgesamt 27 Merkmalen, wobei die fünf Merkmale spatial, temporal, kausal, modal und Vorhandensein in einer Merkmalshierarchie organisiert sind, also Unterkategorien besitzen. Die Unterkategorien für temporale Merkmale entstammen der Analyse von Durrell und Brée (1993), die Untermerkmale der Kategorie spatial der Analyse von Schröder (1986) - zu Vorbandensein vgl. die Abschnitte 4.3 und 5.

- Annotatoren bewegen sich mittels Entscheidungsbaumverfahren durch die jeweiligen Hierarchien und haben auch die Möglichkeit, anstelle eines maximal spezifischen Interpretationstyps ein Hyperonym als Interpretation anzugeben. In den Analysen in Abschnitt 4 machen wir nur von den jeweiligen Obertypen Gebrauch.

- Zusätzlich zu den fünf Bedeutungen mit Unterbedeutungen gibt es 22 Bedeutungen ohne Unterbedeutung: Zustand, komitativ, Quantitätsdiffereñ, Beteiligung, Unterordnung, Zuordnung, Wechselberiehung, Rangfolge, Über-/Unterscbreitung, Bequgspunkt, Thema, Empfänger, Stellvertretung/Ersatr, Austausch, komparativ, restriktiv, kopulativ, adversativ, distributiv, Stellungnahme, Agens, Realisation. Im Gegensatz zu den Merkmalen mit Untertypen mögen viele der hier genannten Merkmale eher abstrakt erscheinen. Wir werden die Merkmale anhand der Fallstudien in Abschnitt 4 genauer erläutern.

- Es besteht die Möglichkeit der Kreuzklassifikation, so dass bestimmte Eigenschaften der Interpretation nicht als Merkmale dargestellt werden; dies gilt insbesondere für die Repräsentation direktionaler Interpretationen. Direktionalität sollte bei sämtlichen lokalen Bedeutungsvarianten verfügbar sein, würde aber bei der Mehrzahl von Präpositionen mit einer lokalen Interpretation zu einer vorhersagbaren Hinzufügung des Merkmals direktional führen, das zudem nahezu immer aus dem Kasus des Objekts abgeleitet werden kann.

- Pro Präposition liegen zwischen einer und siebzehn Bedeutungen vor.

Das Vorkommen der Präpositionen im NZZ-Korpus in PNKen und PPen (PPen mit $\mathrm{N}$ aus PNK und PPen mit $\mathrm{N}$ nicht in PNK) ist im Folgenden dargestellt; die Präpositionen sind hierbei nach ihrer Polysemie geordnet:

\begin{tabular}{|l|c|c|r|r|r|}
\hline & $\begin{array}{c}\text { Bedeu- } \\
\text { tungen }\end{array}$ & $\begin{array}{c}\text { Unterbedeu- } \\
\text { tungen }\end{array}$ & PNK & $\begin{array}{c}\text { PP } \\
\text { (N in PNK) }\end{array}$ & $\begin{array}{c}\text { PP } \\
\text { (N nicht in PNK) }\end{array}$ \\
\cline { 1 - 5 } gemäß & 1 & 1 & 455 & 418 & 177 \\
\cline { 1 - 1 } seit & 1 & 2 & 297 & 1.298 & 1.065 \\
\cline { 1 - 1 } während & 1 & 3 & 24 & 356 & 2.507 \\
\cline { 1 - 1 } hinter & 1 & 8 & 61 & 343 & 1.532 \\
\cline { 1 - 1 } dank & 2 & 3 & 171 & 246 & 741 \\
\cline { 1 - 1 } mittels & 2 & 3 & 195 & 75 & 154 \\
\hline
\end{tabular}




\begin{tabular}{|c|c|c|c|c|c|}
\hline & $\begin{array}{l}\text { Bedeu- } \\
\text { tungen }\end{array}$ & $\begin{array}{l}\text { Unterbedeu- } \\
\text { tungen }\end{array}$ & PNK & $\begin{array}{c}\text { PP } \\
(\mathrm{N} \text { in } \mathrm{PNK})\end{array}$ & $\begin{array}{c}\text { PP } \\
\text { (N nicht in PNK) }\end{array}$ \\
\hline wegen & 2 & 3 & 1.091 & 886 & 1.409 \\
\hline neben & 3 & 4 & 164 & 253 & 1.790 \\
\hline durch & 5 & 6 & 1.970 & 7.674 & 6.311 \\
\hline vor & 5 & 15 & 786 & 8.756 & 4.688 \\
\hline bei & 5 & 24 & 2.340 & 11.679 & 6.280 \\
\hline $\mathrm{um}$ & 6 & 7 & 701 & 5.549 & 6.765 \\
\hline ohne & 6 & 11 & 4.632 & 473 & 218 \\
\hline gegen & 6 & 16 & 1.687 & 4.831 & 3.857 \\
\hline an & 6 & 29 & 2.916 & 22.563 & 11.174 \\
\hline nach & 7 & 11 & 5.364 & 18.942 & 4.750 \\
\hline in & 7 & 20 & 20.425 & 93.434 & 16.871 \\
\hline über & 7 & 17 & 1.168 & 9.880 & 9.275 \\
\hline auf & 7 & 29 & 27.011 & 59.938 & 16.131 \\
\hline für & 8 & 10 & 3.885 & 31.029 & 11.612 \\
\hline unter & 10 & 22 & 1.864 & 3.605 & 1.497 \\
\hline mit & 11 & 18 & 13.981 & 43.833 & 13.262 \\
\hline Summe & & & 91.188 & 326.061 & 122.066 \\
\hline
\end{tabular}

Tab. 1: Vorkommen von PNK und PP

Tabelle 1 verdeutlicht, dass nicht alle Präpositionen zwingend polysem sind - und somit eine P-selektionale Analyse, die in jedem Fall die Polysemie der Präposition voraussetzt, nicht tragen würde. Die nicht-polysemen Präpositionen sind gemäß und wäbrend. Die größte Gruppe besteht aus Präpositionen, die zwischen zwei und sechs Bedeutungen besitzen. Schließlich gibt es auch eine Gruppe hochpolysemer Präpositionen, die sieben und mehr Interpretationen besitzen.

Betrachtet man die Gruppe bis zur Präposition neben (mit drei Bedeutungen), so ließe sich der Schluss formulieren, dass niedrige Polysemie auf der Typ-Ebene auch ein geringeres Vorkommen der Präposition nach sich zieht. Diese Annahme bestätigt sich allerdings nicht, wenn die weiteren Präpositionen betrachtet werden. So gehört unter zu den hochpolysemen Präpositionen, es werden aber verhältnismäßig wenig PNKen/PPen mit unter realisiert. Die grafische Darstellung der Tabelle 1 in Abbildung 1 verdeutlicht dies nochmals. Die erste Reihe markiert hier die Häufigkeit der PNKen, die zweite die der PPen mit denselben Substantiven und die dritte die der PPen mit anderen zählbaren Substantiven. 


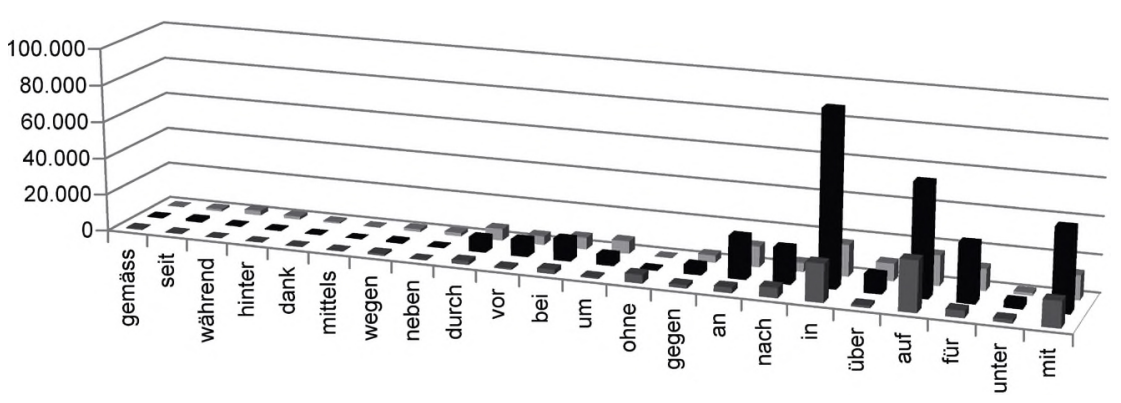

Abb. 1: Vorkommen von PNK und PP (dunkelgrau: PNK, schwarz: PP mit N in PNK, hellgrau: PP mit N nicht in PNK)

Die Präposition obne besitzt die Besonderheit, dass hier mehr Vorkommen in PNKen zu beobachten sind als in PPen.

\section{Pilotstudie: Bedingungen für die Realisation artikelloser Phrasen als Komplemente von obne und unter}

\subsection{Grundannahmen}

Auch eine Analyse, die auf dem Annotation Mining aufsetzt, kann nicht gänzlich unabhängig von introspektiven Urteilen durchgeführt werden. Allerdings kommt introspektiven Bewertungen hier eine indirekte und vermittelnde Rolle zu. Annotation Mining bedeutet ja nichts anderes, als Rohdaten entweder automatisch oder manuell mit Annotationen zu versehen, was wiederum voraussetzt, dass für die relevanten Ebenen linguistische Annotationsschemata entwickelt werden. Es ist nun sehr unwahrscheinlich, dass die Entwicklung eines solchen Schemas - das ja eigentlich eine Mikrogrammatik ist - ohne jedes introspektive Urteil erfolgt. Die Zuordnung einzelner Elemente zu einem Schema kann ohne introspektive Befragung und insbesondere ohne einen bereits vorliegenden Kriterienkatalog nicht erfolgen. Ein zweiter Faktor betrifft die relative Unabhängigkeit der Annotationsschemata. Diese werden typischerweise unabhängig voneinander und auch von unterschiedlichen Personen entwickelt. Somit werden hier introspektive Urteile, wenn diese bei der Entwicklung eine Rolle spielten, auf eine breite Basis gestellt. Gerade vor dem Hintergrund dieser Überlegungen ist das Annotation Mining das Mittel der Wahl, wenn es um explorative Analysen geht. Die einleitenden Überlegungen haben gezeigt, dass PNKen regelhaft sind. Die explorative Analyse dient nun dazu, möglichst objektiv (d.h. auf eine breite empirische Basis gestützt) die Bedingungen dieser Regelhaftigkeit zu identifizieren. Insbesondere, wenn es sich um multikausale Bedingungen handelt, ist ein solches Vorgehen einer traditionellen, durch Introspektion geleiteten Analyse überlegen. 
Für den vorliegenden Fall bieten sich nun Analysetechniken an, die es gestatten, die zugrunde liegende Fragestellung möglichst genau nachzuvollziehen. Eine solche Analysetechnik ist die binäre logistische Regression (Harrell 2001). Die Verletzung der Dudenregel 442 erfährt dabei die folgende Deutung: Die Realisation eines Artikels darf als regelhafte Grundbedingung gelten, d.h. wenn keine anderen Bedingungen erfüllt sind, ist die Realisation des Artikels der Normalfall. Wir betrachten den Wegfall des Artikels als vorherzusagende Eigenschaft und verwenden die im Annotation Mining identifizierten Merkmale von PNKen und PPen als Prädiktoren. Eine binäre logistische Regression identifiziert nun in den Merkmalen solche, die die Wahrscheinlichkeit eines Artikelwegfalls anheben und solche, die eine Anhebung absenken, so dass insgesamt eine Wahrscheinlichkeit für den Artikelwegfall in Abhängigkeit der vorhandenen Eigenschaften formuliert werden kann.

In den Analysen ist somit die abhängige Variable der Faktor DET, der die beiden Werte no und yes annehmen kann. Die folgenden Eigenschaften werden durch 92 Merkmale repräsentiert:

- Welche Interpretation besitzt die Präposition?

- Zu welchen Wortfeldern wird das Substantiv gezählt?

- Welche interne syntaktische Struktur besitzt das nominale Komplement: Wird es adjektivisch modifiziert, besitzt es ein Komplement, welche Kategorie besitzt das Komplement?

- Handelt es sich beim Kopf des nominalen Komplements um ein Kompositum oder nicht?

- Handelt es sich beim Nomen um ein abgeleitetes Nomen?

- Welche externe syntaktische Einbettung für die PNK/PP liegt vor: Handelt es sich um einen adverbialen Modifikator oder ein regiertes Komplement?

Bevor die Daten auf diese Weise analysiert wurden, war zunächst eine Bereinigung der Daten erforderlich: PNKen, die in Überschriften, Titeln und Zitaten vorkamen, wurden ebenso wenig für die Analyse berücksichtigt wie idiomatische Ausdrücke. Der Grund für die Elimination von PNKen in Überschriften, Titeln und Zitaten ist, dass diese Textstrukturen die extragrammatische Verknappung eines Textes in besonderem Maße fördern und somit hier auftretende PNKen möglicherweise eine grammatische Analyse verzerren würden. Ähnliches gilt auch für idiomatische Ausdrücke, die die Form einer PNK, aber ebenso häufig auch die Form einer PP annehmen. Deswegen wurden auch sie eliminiert. Insgesamt liegen den beiden Pilotanalysen die Daten in Tabelle 2 zugrunde: ${ }^{7}$

Die Daten in Tabelle 2 unterscheiden sich nicht nur deswegen von den Daten aus Tabelle 1 , weil PNKen in bestimmten Textstrukturen nicht berücksichtigt worden sind. Weiterhin aus- 


\begin{tabular}{|l|c|c|c|}
\hline Präposition & $\Sigma$ & PP & PNK \\
\hline ohne & 3.750 & 591 & 3.159 \\
\hline unter & 5.181 & 4.334 & 857 \\
\hline
\end{tabular}

Tab. 2: Artikelrealisation bei den Präpositonen ohne und unter

Zur Bewertung der eigentlichen Analyse ist es zunächst erforderlich, eine automatische Klassifikation durchzuführen, die davon ausgeht, dass alle Merkmale, die relevant sein könnten, auch tatsächlich relevant sind (einen

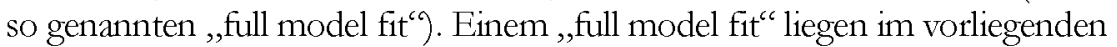
Fall 92 Merkmale zugrunde, die die oben genannten Eigenschaften abbilden. Auf der Basis dieses ,full model fits" werden Merkmale, deren Relevanz als gering eingeschätzt wird, entweder automatisch oder manuell verworfen und darauf erneut ein Modell entwickelt. Ein Merkmal wird dann verworfen, wenn es einen Modellkoeffizienten besitzt, der sich mit einer bestimmten Wahrscheinlichkeit nicht von 0 unterscheidet. Wenn ein Modellkoeffizient (= Gewichtung des Klassifikationsmerkmals) den Wert 0 besitzt, dann bedeutet dies nichts anderes, als dass das betreffende Merkmal für die vorliegende Analyse keine Bedeutung besitzt. Bei einer automatischen Analyse, etwa durch „fast backward elimination", geht man davon aus, dass nur Merkmale erhalten bleiben, für deren Koeffizienten mit einer bestimmten Wahrscheinlichkeit (typischerweise wenigstens 95\%) angenommen werden kann, dass sie von 0 verschieden sind (vgl. Bayyen 2008, S. 186). Dies kann aber dazu führen, dass einflussreiche Merkmale eliminiert werden, weswegen Harrell (2001, S. 56) auch von einem automatischen Verfahren abrät. Als Faustregel kann man angeben, dass ein Prädiktor entfernt wurde, wenn die Wahrscheinlichkeit, dass der Wert ungleich 0 ist, niedriger lag als 20\%. Für Prädiktoren, die nicht in den Bereich zwischen 20 und $95 \%$ fielen, wurde jeweils ein Modell ohne und mit dem Prädiktor konstruiert und die Modellparameter wurden miteinander verglichen.

\subsection{Binäre logistische Regression}

Für die binäre logistische Regression gehen wir davon aus, dass es eine dichotome abhängige Variable gibt, beispielsweise eine Kategorie mit zwei

geschlossen werden automatisch, aber falsch annotierte Daten (etwa aufgrund von Tippfehlern), unvollständige Sätze, Postpositionen, die falsch als P gechunkt wurden, Zählbarkeitsfehler wegen Synkretismus, nicht erkannter Pluralform oder nominalisiertem Infinitiv, PPen in Funktionsverbgefügen, PP mit postnominalem Adverb, Verbpartikel, die fälschlich als Präposition klassifiziert und eine fehlerhafte syntaktische Analyse nach sich ziehen, sowie Numerativkonstruktionen. 
Werten, die intern durch die Werte 0 und 1 kodiert werden kann. Die unabhängigen Variablen können metrische oder kategoriale Werte besitzen. Im vorliegenden Modell ist die abhängige Kategorie die Zugehörigkeit zu den Kategorien PNK und PP; die unabhängigen Variablen besitzen kategoriale Werte, aus denen wir ableiten wollen, ob ein Artikel realisiert wird (= PP) oder nicht $(=\mathrm{PNK})$. Es sind die in Abschnitt 4.1 vorgestellten Merkmale.

Die binäre logistische Regression bestimmt allerdings nicht, ob eine Phrase zu einer der beiden Kategorien gehört, sondern gibt die Wahrscheinlichkeit an, mit der die Phrase zu den genannten Kategorien gehört. Man spricht hier von logistischer Regression, weil im Gegensatz zur linearen Regression nicht die gewichtete Summe der Prädiktoren gebildet wird, sondern die logistische Funktion der gewichteten Summe der Prädiktoren: $\log i t(p)=\log (p / 1-p)$. Die gewichtete Summe der Prädiktoren kann Werte zwischen $+\infty$ und $-\infty$ annehmen. Die inverse logit-Funktion bildet diese Werte aber auf das Intervall $[0 ; 1]$ ab und stellt somit sicher, dass die resultierende Funktion tatsächlich eine Wahrscheinlichkeitsfunktion ist, die eine S-Form annimmt. Im Modell liegt somit eine Abbildung von Eigenschaften auf 92 Merkmale vor, von denen diejenigen Merkmale als Prädiktoren verwendet werden, die die Chancen beeinflussen, dass ein Vorkommen der einen oder anderen Kategorie zugeordnet wird.

In den folgenden Modellen wird jeweils die Wahrscheinlichkeit für die Realisation eines Artikels bestimmt. Die unabhängigen Variablen (= Prädiktoren) können hierbei positive und negative Werte annehmen. Negative Werte sprechen gegen die Realisation eines Artikels, positive Werte für die Realisation. Betrachten wir ein vereinfachtes Beispiel, in dem es nur einen Prädiktor gibt, der den Wert -1,386 annimmt. Für einen solchen Wert ergibt die Anwendung des inversen logit den Wert 0,2, d.h. die Wahrscheinlichkeit für die Weglassbarkeit des Artikels liegt in der betreffenden Konstruktion bei $1-0,2=0,8$.

\subsection{Logistische Modelle für den Wegfall des Artikels bei obne und unter}

Das in Abschnitt 4.1 beschriebene Verfahren der Elimination von Prädiktoren führte zu Modellen für den Wegfall des Artikels bei den Präpositionen ohne und unter, die auf 13 (für ohne) bzw. 22 (für unter) Merkmalen aus den ursprünglichen 92 Merkmalen basieren.

\subsubsection{Das Modell für obne}

Wir betrachten zunächst das Modell für obne, das in Tabelle 3 dargestellt wird. 


\begin{tabular}{|l|c|c|c|}
\hline & Koeffizient & s.E. & p \\
\hline ACHSENABSCHNIT'T & $-2,40$ & 0,11 & 0,000 \\
\hline UNG-NOMINAIISIERUNG & $-1,36$ & 0,19 & 0,000 \\
\hline ADJ IN N' & 1,14 & 0,12 & 0,000 \\
\hline KAUSAL & 1,21 & 0,13 & 0,000 \\
\hline KOMITATIV & 2,28 & 0,52 & 0,000 \\
\hline BETEILIGUNG & 3,40 & 0,49 & 0,000 \\
\hline VORHANDENSEIN & $-0,78$ & 0,15 & 0,000 \\
\hline DEP-S & 5,08 & 1,05 & 0,000 \\
\hline DEP-NP & 2,97 & 0,17 & 0,000 \\
\hline DEP-PP & 2,20 & 0,15 & 0,000 \\
\hline GN-RELATION & $-1,03$ & 0,41 & 0,011 \\
\hline GN-ATTRIBUT & $-1,35$ & 0,30 & 0,000 \\
\hline GN-EREIGNIS & $-0,84$ & 0,14 & 0,000 \\
\hline GN-ARTEFAKT & $-0,41$ & 0,16 & 0,008 \\
\hline
\end{tabular}

Tab. 3: Koeffizienten für ein logistisches Modell für den Wegfall des Artikels bei obne

Tabelle 3 gibt die relevanten Faktoren mit ihren Koeffizienten, d.h. Gewichtungen an, den Standardfehler der Schätzung (engl. ,standard error", d.h. S.E.) und die Wahrscheinlichkeit, dass die Gewichtung nur zufällig von 0 abweicht. Der Standardfehler kann genutzt werden, um nach der Daumenregel (Koeffizienz \pm S.E. $\times 2$ 2) 95-\%-Konfidenzintervalle für die Koeffizienten zu bestimmen. Selbst bei den Werten für GN-RELATION und GNARTEFAKT macht diese Berechnung deutlich, dass die Konfidenzintervalle 0 nicht einschließen.

Würden die Intervalle den Wert 0 einschließen, dann wäre es durchaus denkbar, dass der Wert eines Koeffizienten tatsächlich 0 beträgt, was bedeuten würde, dass dieser Koeffizient für den Wegfall eines Artikels keine Rolle spielt. Der Wert für den Achsenabschnitt kann in jedem Fall vernachlässigt werden: Er ergibt sich als Artefakt aus der Modellierung der jeweils binären Werte für die unabhängigen Variablen und der Form der internen Kodierung dieser Werte.

Die identifizierten Eigenschaften können wie folgt interpretiert werden: Die Merkmale KAUSAL, KOMITATIV und BETEILIGUNG (zur Definition dieser Eigenschaften siehe Abschnitt 5) besitzen positive Werte. Sie verschieben somit die Gewichte zugunsten einer Realisation eines Artikels. Eine erste naheliegende Interpretation dieser Werte könnte lauten: Wenn 
eine Präposition die Merkmale KAUSAL, KOMITATIV oder BE'TEILIGUNG besitzt, dann ist die Realisation einer NP (mit Artikel) wahrscheinlicher als die Realisation einer Nominalprojektion ohne Artikel. Beispiele für die Zuweisungen dieser Interpretationen sind in (27) bis (29) gegeben. ${ }^{8}$

(27) KAUSAL:

a. Sämtliche kurdischen Politiker sind davon überzeugt, dass ohne einen Machtwechsel in Bagdad die Kurdenfrage des Iraks nicht zu lösen sei. (NZZ, AUSLAND, 28.01.1993)

b. Und für eigentliche Verhandlungen hat Frau Kumaratunga nach eigenen Aussagen obne absolute Parlamentsmehrbeit noch kein klares Mandat. (NZZ, AUSLAND, 14.09.1994)

(28) KOMITATIV:

a. Ein mobiles Einsatzkommando überwältigte nach Polizeiangaben den aus Tunesien stammenden Geiselnehmer, als er ohne das Kind den Gerichtssaal verließ. (NZZ, VERMISCHTE MELDUNGEN, 14.07.1993)

b. Kessler, der ohne Anwalt vor Gericht erschien, betonte in seinem umfangreichen Plädoyer, seine anerkanntermaßen scharfe Kritik richte sich nicht gegen die Juden als Religionsgemeinschaft.

(NZZ, ZÜRICH UND REGION, 11.03.1998)

(29) BETEILIGUNG:

a. Die abschließende Verhandlung der Nevada State Athletic Commission fand obne den Angeklagten statt, dem außerdem die Kosten des Verfahrens aufgebrummt wurden. (NZZ, SPORT, 10.07.1997)

b. (keine Beispiele im Korpus gefunden)

Die Beispiele verdeutlichen - zumindest für die Merkmale KAUSAL und KOMITATIV, dass die Bedingungen für die Realisation nicht absolut zu werten sind, und insbesondere auch nicht isoliert. Ansonsten dürften die Belege (27b) und (28b) gar nicht existieren.

Das Merkmal VORHANDENSEIN besitzt demgegenüber einen negativen Wert. Liegt diese Interpretation vor, dann ist die Wahrscheinlichkeit der Realisation einer Nominalprojektion ohne Artikel höher als die Wahrscheinlichkeit der Realisation einer NP (mit Artikel). Beispiele finden sich in (30).

(30) VORHANDENSEIN:

a. Was weiter als positives Zeichen zu deuten wäre, Fragezeichen betreffend Stabilität und Solidität gegen die Portugiesen zum Trotz, ist die Serie von acht Partien obne Niederlage, die längste seit 1924. (NZZ, SPORT, 02.04.1993)

Die Interpretation KAUSAL subsumiert die Interpretation KONDITIONAL, die in Beispiel (27a) vorliegt. 
b. Die Anklage wirft dem ersten von drei Angeklagten, einem 32jährigen Mann obne Beruf, die Mitwirkung an allen drei Tötungsdelikten vor. (NZZ, STADT UND KANTON ZÜRICH, 14.04.1993)

Wir sehen hier also zwei unterschiedliche Interpretationsgruppen für die Präposition ohne. Besitzt die Präposition die Interpretationen KAUSAL, KOMITATIV oder BETEILIGUNG, wird die Realisation eines Artikels bevorzugt. Bei der Interpretation VORHANDENSEIN hingegen wird die Weglassbarkeit des Artikels suggeriert. In Abschnitt 5 werden wir auf diese Verteilung zurückkommen und fragen, ob die hier dargestellte Verteilung der Interpretationen der Präposition obne nicht eine zugrunde liegende Eigenschaft dieser Konstruktion verbirgt.

Die mit DEP beginnenden Merkmale spezifizieren syntaktische Komplemente des Nomens in einer PNK, d.h. selegierte Sätze, postnominale NPen und postnominale PPen. Die drei Merkmale suggerieren, dass die Realisation eines Komplements die Realisation eines Artikels fördert. Das Merkmal ADJ IN N' legt fest, ob das Nomen in einer PNK adjektivisch modifiziert wurde. Das Merkmal besitzt wie DEP-S, DEP-NP und DEP-PP einen positiven Koeffizienten, d.h. fördert die Realisation eines Artikels. Allen vier Merkmalen ist gemein, dass sie die syntaktische Komplexität der Nominalprojektion erhöhen, so dass man den Schluss ziehen könnte, dass eine höhere syntaktische Komplexität der Nominalprojektion auch die Wahrscheinlichkeit eines Artikelwegfalls deutlich absenkt. Wir werden aber bei der Analyse von unter sehen, dass eher die Realisation eines Komplements als die syntaktische Komplexität die Wahrscheinlichkeit eines Artikelwegfalls beeinflusst.

Deverbale Nomina, die mit -ung gebildet werden, unterstützen ebenfalls den Wegfall des Artikels. Hier bedarf es einer genaueren Untersuchung bzw. auch der Integration von Verfahren zur Disambiguierung ung-nominalisierter Substantive, so wie dies etwa in Eberle (2009) vorgeschlagen wird. Es ist allerdings festzuhalten, dass eine Präferenz des Artikelwegfalls bei deverbalen Nomina eine übergreifende Eigenschaft zu sein scheint - sie begegnet uns erneut im Modell für unter.

Bei den Merkmalen GN-RELATION, GN-ATTRIBUT, GN-EREIGNIS und GN-ARTEFAKT handelt es sich um eine Zuordnung der Substantive zu Wortfeldern aus GermaNet (vgl. Kunze/Lemnitzer 2002). Diese Merkmale bezeichnen also semantische Eigenschaften der Substantive. Nomina, die mehr als einem Wortfeld angehören, sind polysem oder homonym. Für solche Substantive wird in der vorliegenden Klassifikation keine Disambiguierung durchgeführt.

Da die Zuordnung typbasiert erfolgt, besitzt ein mehrdeutiges Nomen in jeder PNK bzw. PP jede Zuordnung aus GermaNet. Die Definition der für das Modell für ohne relevanten Kategorien in WordNet ist in (31) gegeben. 
(31) a. relation: an abstraction belonging to or characteristic of two entities or parts together.

b. attribute: an abstraction belonging to or characteristic of an entity.

c. event: something that happens at a given place and time.

d. artefact: a man-made object taken as a whole.

Beispiele für Substantive des Wortfelds relation sind in (32a) gegeben, Beispiele des Wortfelds attribute in (32b), des Wortfelds event in (32c) und des Wortfelds artefact in $(32 \mathrm{~d})$.
a. Kameradschaft, Heiratsantrag
b. Tonhöhe, Gefühllosigkeit
c. Diffusion, Konjunkturprogramm
d. Klosteranlage, Schutzvorrichtung

Diese Wortfelder unterstützen den Wegfall des Artikels, was erneut am negativen Vorzeichen erkennbar ist. Dies ist auch deswegen interessant, weil unter diese Wortfelder keineswegs nur Abstrakta fallen, die in der Literatur ebenso oft wie fälschlich den Massentermen zugeordnet werden (vgl. etwa Bale/Barner 2009), sondern auch Konkreta. Dass diese Annahme nicht korrekt sein kann, sieht man an Substantiven wie Gefühllosigkeit oder Kameradschaft, die jeweils als zählbar klassifiziert werden können.

Diese Merkmale können als Selektionsrestriktionen interpretiert werden für substantivische Komplemente von obne, wenn obne die Interpretation VORHANDENSEIN besitzt. Bei mehr als 50\% der Vorkommen von ohne mit der Interpretation VORHANDENSEIN wird ein Substantiv ausgewählt, das in wenigstens eines der oben genannten Wortfelder fällt, wobei 51,5\% der Substantive das Merkmal GN-EVENT besitzen und nur 35,3\% der Substantive das Merkmal GN-ARTEFACT.

\subsubsection{Das Modell für unter}

In Tabelle 4 findet sich das Modell für unter, das mit seinen 22 Merkmalen nahezu doppelt so viele relevante Merkmale aufweist wie das Modell für ohne. Dass hier doppelt so viele Merkmale erforderlich sind, liegt sicherlich insbesondere daran, dass unter wesentlich mehr Bedeutungen als obne aufweist und damit mehr Interpretationen der Präposition und auch mehr Wortfelder der Substantive berücksichtigt werden müssen.

Hier fällt nun allerdings als erstes auf, dass alle Koeffizienten für die Präpositionsbedeutungen ein positives Vorzeichen besitzen. Es werden zwar fünf der zehn Oberbedeutungen von unter im Modell berücksichtigt, aber diese Oberbedeutungen legen jeweils eine Blockade des Artikelwegfalls nahe. Es gibt keine Bedeutungen, die unmittelbar für den Artikelwegfall stehen, so wie etwa VORHANDENSEIN für ohne. Hier zeigt sich ein Muster, dass 
ich in Kiss (2007) bereits für spatiale Interpretationen beobachtet habe. In Kiss (ebd.) stellte ich fest, dass spatiale Interpretationen bei PNKen mit unter sehr selten auftreten, um nicht zu sagen nie. Vergleicht man den Koeffizienten des Merkmals SPATIAL mit den Werten für die anderen Bedeutungen, so wird erwartet, dass insbesondere UNTERORDNUNG, ZUORDNUNG und ÜBER-/UNTERSCHREITUNG die Realisation von PNKen blockieren. Wir finden also weniger lizenzierende Merkmale für den Artikelwegfall als Bedeutungen, die den Wegfall behindern.

\begin{tabular}{|l|c|c|c|}
\hline & Koeffizient & S.E. & p \\
\hline ACHSENABSCHNITT & $-0,4379$ & 0,1657 & 0,008 \\
\hline UNG-NOMINALISIERUNG & $-0,8346$ & 0,2259 & 0,000 \\
\hline ADJ IN N' & $-1,0177$ & 0,1432 & 0,000 \\
\hline KOMPOSITUM & 2,1719 & 0,2538 & 0,000 \\
\hline REGIERT & 1,9894 & 0,3017 & 0,000 \\
\hline SPATIAL & 2,3237 & 0,2044 & 0,000 \\
\hline KAUSAL & 1,3047 & 0,2272 & 0,000 \\
\hline UNTERORDNUNG & 3,0529 & 0,2559 & 0,000 \\
\hline ZUORDNUNG & 3,4228 & 0,1861 & 0,000 \\
\hline ÜBER-/UNTERSCHREITUNG & 4,4186 & 0,3677 & 0,000 \\
\hline DEP-S & 8,4717 & 4,0734 & 0,037 \\
\hline DEP-NP & 0,8551 & 0,1436 & 0,000 \\
\hline DEP-PP & 0,3043 & 0,2170 & 0,161 \\
\hline GN-GRUPPE & 0,5241 & 0,2563 & 0,041 \\
\hline GN-KOMMUNIKATION & $-0,9149$ & 0,1443 & 0,000 \\
\hline GN-ORT & 2,2704 & 0,6208 & 0,000 \\
\hline GN-RELATION & $-2,1161$ & 0,6022 & 0,000 \\
\hline GN-BESITZ & $-0,8482$ & 0,3665 & 0,021 \\
\hline GN-ATTRIBUT & $-2,2847$ & 0,2741 & 0,000 \\
\hline GN-ARTEFAKT & 0,4169 & 0,1601 & 0,009 \\
\hline GN-MENSCH & 1,8870 & 0,4999 & 0,000 \\
\hline HL-AD & $-1,0253$ & 0,1888 & 0,000 \\
\hline HL-AS & $-1,4214$ & 0,3804 & 0,000 \\
\hline
\end{tabular}

Tab. 4: Koeffizienten für ein logistisches Modell für den Wegfall des Artikels bei unter 
Einige Muster aus dem Modell für obne sind auch im Modell für unter wieder vorhanden. Insbesondere ist beobachtbar, dass die Merkmale DEP-S, DEPNP und DEP-PP erneut gegen den Wegfall des Artikels sprechen. ${ }^{9}$ Das Merkmal ADJ IN N' besitzt bei unter allerdings einen negativen Koeffizienten. Dies bedeutet, dass das Vorhandensein von Adjektiven in nominalen Komplementen von obne die Weglassbarkeit des Artikels erschwert, dieselbe Bedingung bei Komplementen von unter aber den Wegfall des Artikels begünstigt.

Darüber hinaus scheint zunächst auch die externe Distribution der Phrase eine Rolle zu spielen: Das Merkmal REGIERT bestimmt, ob die PP bzw. PNK von einem Substantiv oder Verb regiert wird. Es spielt bei obne keine Rolle, während es bei unter den Wegfall des Artikels erschwert. Der Grund hierfür kann aber auch sein, dass es nur sehr wenige Verben oder Nomina gibt, die die Präposition obne regieren. Präpositionalobjekte, deren Kopf die Präposition unter ist, sind dagegen nicht so selten. Im vorliegenden Korpus werden nur 1,2\% der Vorkommen von ohne überhaupt regiert, aber $3,6 \%$ der Vorkommen von unter, wobei unter ja auch insgesamt häufiger vorkommt. Dennoch kann man festhalten, dass Präpositionalobjekte mit unter den Wegfall des Artikels nicht unterstützen.

Weiterhin können wir beobachten, dass nominale Komplemente von unter, die als Komposita analysiert werden können, den Wegfall des Artikels ebenfalls nicht unterstützen. Es wird allerdings weiterer Untersuchungen bedürfen, um herauszufinden, warum dies so ist. Schließlich erkennen wir beim Modell für unter ebenso wie beim Modell für obne den Einfluss der Nominalisierung durch -ung. Nominalisierungen unterstützen den Wegfall des Artikels.

Die einzelnen Merkmale für die Wortfelder des Substantivs zeigen ein heterogenes Bild, das zu weiteren Untersuchungen einlädt: Es ist zunächst einmal auffällig, das GN-ORT einen hohen positiven Wert besitzt. GN-ORT dürfte Ausdruck einer Selektionsrestriktion bei spatialen Interpretationen von unter sein. Andere GN-Merkmale unterstützen hingegen den Wegfall des Artikels. Das Modell für unter macht aber nicht nur Gebrauch von Wortfeldern aus GermaNet, sondern auch von der ontologischen Klassifikation in HaGenLex. Die beiden relevanten Merkmale HL-AS (HaGenLex, abstrakt und statisch) und HL-AD (HaGenLex, abstrakt und dynamisch) sind Beschreibungen für abstrakte dynamische bzw. abstrakte statistische Sachverhalte aus der Sortenhierarchie von HaGenLex (vgl. Hartrumpf et al. 2003). Beispiele für die beiden Gruppen finden sich in (33).

Die Irrtumwahrscheinlichkeit bei DEP-PP liegt oberhalb von 0,05. Dieser Faktor wurde dennoch weiter im Modell gehalten, weil sich die Modellparameter insgesamt verschlechtern, wenn auf diesen Faktor verzichtet wird, vgl. Harrell (2001, S. 56). 
(33) a. abstrakte dynamische Sachverhalte (Merkmal HL-AD): Lauf, Diebstahl, Veränderung

b. abstrakte statistische Sachverhalte (Merkmal HL-As): Umstand, Notlage, Auslastung

Wie auch anhand der Beispiele in (38) erkennbar ist, liegen abstrakte Nomina vor, die aber zählbar sind. Wir sehen somit einen weiteren Grund, an der Annahme zu zweifeln, dass Abstrakta nicht zählbar sein dürfen. Substantive, die als abstrakte statische oder dynamische Konzepte in HaGenLex charakterisiert sind, fördern den Artikelwegfall bei unter.

\subsection{Die Güte der Modelle}

Die Güte logistischer Modelle kann einerseits anhand bestimmter Kennzahlen bemessen werden, andererseits auch durch unterschiedliche Evaluationsverfahren bestimmt werden. Harrell (2001) empfiehlt für die Evaluation logistischer Modelle die so genannte „bootstrap validation“, die im Gegensatz zu anderen Validierungsverfahren, insbesondere im Gegensatz zur „crossvalidation " den Vorteil hat, dass alle Daten zum Training und zum Testen verwendet werden können. Mittels „,bootstrap validation“" kann bestimmt werden, ob das Modell zu sehr an den gesehenen Daten orientiert ist (ein ,overfit ${ }^{\text {cc }}$ vorliegt) oder ob hier plausibel von gesehenen auf nicht gesehene Daten generalisiert werden kann und somit ein Modell mit Vorhersagekraft vorliegt. Bei einer „bootstrap validation“ wird aus einem Datensatz mit $n$ unterschiedlichen Elementen durch Auswahl mit Zurücklegen eine Menge von Datensätzen mit $m(=n)$ nicht-unterschiedlichen Elementen gebildet. Eine Auswahl mit Zurücklegen bedeutet hier, dass ein Datensatz in die Stichprobe übernommen wird, aber auch danach nochmals für die Stichprobe zur Verfügung steht. Die Trainingsmenge enthält also genau so viele Elemente wie der ursprüngliche Datensatz, aber durch das Zurücklegen werden einzelne Daten häufiger als einmal in der Trainingsmenge erscheinen. Die Testmenge besteht dann gerade aus denjenigen Elementen des ursprünglichen Datensatzes, die nie ausgewählt wurden. Ebenso wie bei einer ,crossvalidation " wird dieser Prozess mehrfach wiederholt. Erfreulicherweise legen bereits die reinen Kenndaten der beiden Modelle den Schluss nahe, dass hier Modelle mit Vorhersagekraft entwickelt wurden, und diese Annahme wird auch durch die „bootstrap validation" bestätigt.

\begin{tabular}{|l|c|c|c|c|}
\hline & Model L.R. & $\mathrm{p}$ & $\mathrm{C}$ & $\mathrm{D}_{\text {xy }}$ \\
\hline ohne & $1.063,5$ & 0 & 0,876 & 0,753 \\
\hline unter & $2.245,6$ & 0 & 0,937 & 0,874 \\
\hline
\end{tabular}

Tab. 5: Grundlegende Kenndaten der Modelle 
Der Wert „Model L.R.“ (= „Model Likelihood Ratio") gibt an, wie sehr sich die Vorhersagekraft des Modells von einem Modell mit willkürlich gewählten Prädiktoren (bzw. eben ohne jede Prädiktoren) verändert. Unter ,p" wird erneut die Wahrscheinlichkeit bestimmt, mit der diese Veränderung nur zufällig sein könnte - sie liegt bei 0 .

Besonders relevant sind die hohen Werte für , $\mathrm{C}^{\prime}$ und „Somers $\mathrm{D}_{\mathrm{xy}}$ " in Tabelle 5. Der Somers $D_{x y}$-Wert bestimmt das Verhältnis von konkordanten zu nicht-konkordanten Übereinstimmungen bei einem Modell, dessen abhängige Variable die Werte 0 und 1 annehmen kann. Eine Übereinstimmung ist konkordant, wenn im Modell die vorhergesagten Wahrscheinlichkeiten mit den tatsächlichen Klassenzugehörigkeiten korrespondieren. Der Wert für $D_{x y}$ entspricht $(C-0,5) \times 2$. C und $D_{x y}$ beschreiben eigentlich denselben Sachverhalt, aber $D_{x y}$ ist eine Abbildung des C-Wertes auf eine Wahrscheinlichkeitsskala. Ein willkürliches Modell ohne Vorhersage besitzt einen CWert von $0,5\left(D_{\mathrm{xy}}=0\right)$. Werte für $\mathrm{C}$ über $0,8\left(\mathrm{D}_{\mathrm{xy}}=0,0\right)$ legen den Schluss nahe, dass das Modell Vorhersagekraft besitzt; wir sehen bei dem Modell für unter sogar einen Wert deutlich über 0.9.

Das Ergebnis der „,bootstrap validation" für die beiden Modelle ist in Tabelle 6 dargestellt; hierbei wurden jeweils 200 Iterationen durchgeführt, d.h. 200 Trainingssätze wurden erstellt, mit den Modellparametern trainiert und mit den verbleibenden Daten evaluiert.

a. obne

\begin{tabular}{|l|c|c|c|c|c|}
\hline & Alle Daten & Training & Test & Optimismus & Korrektur \\
\hline $\mathrm{D}_{\mathrm{xy}}$ & 0,7526 & 0,7570 & 0,7500 & 0,0070 & 0,7456 \\
\hline $\mathrm{E}_{\max }$ & 0,0000 & 0,0000 & 0,0096 & 0,0096 & 0,0096 \\
\hline
\end{tabular}

b. unter

\begin{tabular}{|l|c|c|c|c|c|}
\hline & Alle Daten & Training & Test & Optimismus & Korrektur \\
\hline $\mathrm{D}_{\mathrm{xy}}$ & 0,8736 & 0,8744 & 0,8692 & 0,0052 & 0,8684 \\
\hline $\mathrm{E}_{\max }$ & 0,0000 & 0,0000 & 0,0055 & 0,0055 & 0,0055 \\
\hline
\end{tabular}

Tab. 6a und b: „Bootstrap validation" der Modelle

Die Zeilen geben darüber Auskunft, dass der ursprüngliche $\mathrm{D}_{\mathrm{xy}}$-Wert für das Modell für ohne bei 0,7526 lag; die Differenz zwischen dem Training bei der „bootstrap validation“" und dem Test betrug allerdings $(0,757-0,750)=0,007$. Dieser Wert charakterisiert den Optimismus des Modells, d.h. denjenigen Anteil an der Vorhersagekraft, der einer zu starken Ausrichtung an die Trainingsdaten geschuldet ist. Entsprechend muss der ursprüngliche Wert um dieses $\mathrm{Ma} 3$ an Optimismus nach unten korrigiert werden, so dass sich nach Validierung ein Wert von 0,7456 ergibt. Entsprechendes gilt in der zweiten Zeile für die durchschnittliche maximale Fehlerrate. Im Ursprungsmodell 
liegt diese bei 0 , weil mit allen Daten trainiert wurde. Der Testfall zeigt aber eine durchschnittliche Veränderung von 0,0096, so dass dieser Wert nun als Fehlerrate angesetzt wird. Die Ergebnisse für unter wiederholen die für obne.

Vor dem Hintergrund dieser Evaluation erfahren die Koeffizienten der oben genannten Modelle die folgende Interpretation: Je höher ein Wert ausfällt, desto einflussreicher ist der Wert, desto deutlicher wird die Wahrscheinlichkeit für die Weglassbarkeit des Artikels in Richtung des Vorzeichens des Koeffizienten modifiziert. So ist etwa der Wert für DEP-S in beiden Modellen sehr hoch und positiv, so dass wir davon ausgehen können, dass die Realisation eines sententialen Komplements des Nomens die Weglassbarkeit des Artikels extrem erschwert. Liegen aber - etwa bei obne - keine Dependenten des Substantivs vor, dafür aber die entsprechenden Interpretationen von Präposition und Substantiv, dann ist die Weglassbarkeit des Artikels sehr wahrscheinlich. Wir wollen im folgenden Abschnitt der Frage nachgehen, ob es sich nun tatsächlich um rein semantische Faktoren handelt oder hier zugrunde liegende strukturelle Faktoren eine Rolle spielen könnten.

\section{Externe Faktoren beim Artikelwegfall mit obne?}

Eine Interpretation der Präposition obne - VORHANDENSEIN - unterstützt die Weglassbarkeit des Artikels, während die Interpretationen KAUSAL, KOMITATIV und BETEILIGUNG die Weglassbarkeit eher erschweren.

Hier stellt sich die Frage, ob die Verteilung aus intrinsischen Faktoren der Interpretationen erklärt werden kann, oder ob nicht doch andere, verborgene Faktoren eine Rolle spielen könnten. Wenn wir die Interpretationsmöglichkeiten von obne insgesamt betrachten, fällt auf, dass die Interpretationen zumindest in Teilen ein syntaktisches Korrelat besitzen, somit also eine Abbildung zwischen Interpretationen, syntaktischen Korrelaten und der Artikelweglassbarkeit denkbar ist.

Hierzu erscheint es zunächst sinnvoll, die Bedeutung der Präposition ohne zusammen mit der Bedeutung der Präposition mit zu betrachten. Das Annotationsschema für Präpositionsbedeutungen trägt den Besonderheiten dieser beiden Präpositionen - und der Tatsache, dass zumindest einige Interpretationen der einen in Opposition zu Interpretationen der anderen zu charakterisieren sind - dadurch Rechnung, dass bestimmte Kategorien nur von diesen Präpositionen besetzt werden können.

Die Präposition obne teilt mit der Präposition mit die Interpretation VORHANDENSEIN mit den Untertypen SYNTHETISCH und ANALYTISCH. Während diese Interpretation bei mit angibt, dass etwas vorhanden ist, drückt sie bei obne einen Mangel aus. Die Binnendifferenzierung von VORHANDENSEIN, SYNTHETISCH und ANALYTISCH kann leichter anhand von mit erläutert werden: 
VORHANDENSEIN bezeichnet im Schema das Vorhandensein (bzw. bei obne: Fehlen) einer Sache, eines Merkmals oder einer Eigenschaft; SYNTHETISCH bezeichnet das Vorhandensein einer Sache, eines Merkmals oder einer Eigenschaft, die als einer anderen zugehörig betrachtet werden kann, ohne im Sinne einer Mereologie Teil des anderen zu sein; man kann diese Art der Zugehörigkeit auch als eine betrachten, die durch synthetische Urteile erfasst werden kann, z.B. eine Flasche mit Verqierung. Demgegenüber beschreibt ANALYTISCH eine Zugehörigkeit, die tatsächlich als Teil-Ganzes-Beziehung beschrieben werden kann bzw. entsprechend durch ein analytisches Urteil ausgedrückt wird. Die analytische Form der Urteile hat den Charakter des Pleonastischen und deswegen findet man typische analytische Interpretationen von mit nur dann, wenn das Teil in bestimmter Weise modifiziert wird, wie die Kontraste in (34) zeigen:

(34) a. eine Hand mit Fingern vs. eine Hand mit drei Fingern

b. ein Auto mit Motor vs. ein Auto mit Dieselmotor

Für ohne gilt dies natürlich nicht in gleichem Maße, weil das Fehlen eines ansonsten mitgedachten Teils einen höheren Informationsgehalt besitzt als das Vorhandensein dieses mitgedachten Teils:
a. ein Auto ohne Motor
b. eine Hand ohne Daumen

Die drei Merkmale VORHANDENSEIN, SYNTHETISCH und ANALYTISCH können die Präpositionen mit und obne nur annehmen, wenn sie nominale Phrasen modifizieren.

Zusätzlich zu diesen Merkmalen besitzt obne die in (36) aufgeführten Interpretationen:

KAUSAL, KONDITIONAL, ART UND WEISE, BEGLEITUMSTAND, BETEILIGUNG, KOMITATIV, INSTRUMENTAL

Diese Interpretationen treten typischerweise auf, wenn ohne entweder eine verbale Projektion modifiziert oder eine nominale Projektion, deren Kopf eine Ereignisnominalisierung ist. Weiterhin muss hierbei berücksichtigt werden, dass die Merkmale BETEILIGUNG und KOMITATIV ebenso wie das oben bereits angesprochene Merkmal VORHANDENSEIN nur durch die Präpositionen obne und mit besetzt werden kann. Wir können somit sagen, dass zumindest eine Kernbedeutung der Präpositionen ohne und mit durch die Merkmale VORHANDENSEIN, BETEILIGUNG und KOMITATIV bestimmt wird, wobei die Verteilung dieser Merkmale entweder tatsächlich einer externen syntaktischen Distribution (welches Element wird syntaktisch modifiziert) oder einer durch die syntaktische Distribution vermittelten Interpretationsbedingung (wird ein Ereignis oder etwas Dinghaftes modifiziert) geschuldet ist. 
Diese beiden Bedingungen können für die Interpretation BETEILIGUNG wie folgt illustriert werden: In (37) liegen verbale Modifikationen vor, in (38) nominale Modifikationen mit ereignisartigem Charakter.

a. Die Hauptverhandlung wird am 8. Februar notfalls auch ohne den Angeklagten fortgesetzt. (NZZ, AUSLAND, 28.01.1993)

b. Der Viertelmeilen-Sprint, einer der anfänglich vermuteten Höhepunkte, entwickelte sich ohne den großen Abwesenden von Zürich, Michael Johnson, zum erwarteten US-Duell zwischen Antonio Pettigrew und Jerome Young, dem Zweit- und dem Drittschnellsten der Saison. (NZZ, SPORT, 12.08.1999)

(38) a. Nur akademische Miles-Davis-Forscher dürften von diesem detaillierten Einblick in die Werkstatt profitieren und stundenlang abgebrochene Versuche oder gar Durchläufe ohne den Solisten mitverfolgen. (NZZ, PHONO-SPEKTRUM, 02.10.1996)

b. Denn eine Siegerehrung ohne die Amerikanerin ist letztlich doch wohl (noch) ebenso die Ausnahme von der Regel wie die Durchführung einer Abfahrt in zwei Läufen. (NZZ, SPORT, 16.12.1995)

Es scheint also insgesamt so zu sein, dass die einen Interpretationen von ohne nur in verbalen syntaktischen Kontexten realisiert werden können (bzw. in nominalen syntaktischen Kontexten, in denen das Substantiv ereignisartig interpretiert werden muss), während die anderen in nominalen Kontexten realisiert werden. Nominale Kontexte scheinen nun die Artikelweglassbarkeit zu bevorzugen, verbale Kontexte (bzw. Nomina mit Ereignislesart) hingegen blockieren die Artikelweglassbarkeit. Wenn wir nun nochmals das Modell für die Artikelweglassbarkeit bei obne in Tabelle 3 betrachten, so könnte die Präferenz für die Weglassbarkeit bei der Interpretation VORHANDENSEIN bzw. die Präferenz für die Artikelrealisation bei den Interpretationen KAUSAL, KOMITATIV und BETEILIGUNG auch so gedeutet werden, dass die Präposition obne dann ein artikelloses Komplement besitzen kann, wenn die Präposition eine Nominalprojektion modifiziert, aber nicht, wenn sie eine verbale Projektion modifiziert. Dies muss nicht notwendigerweise bedeuten, dass die Analyse der PNK konstruktional sein muss, denn die verbale bzw. nominale Modifikation könnte formal auch durch den semantischen Typ des zu modifizierenden Elements erfasst werden, was dann wiederum auch den Einschluss von Daten wie (38) gestatten würde.

Da nun wiederum die Bedeutungen VORHANDENSEIN, KOMITATIV und BETEILIGUNG diese Verteilung aufweisen und nur als Bedeutungen für die Präpositionen ohne und mit verwendet werden, läge der Schluss nahe, auch das Annotationsschema für die Interpretationen so zu modifizieren, dass etwa das Merkmal [ \pm EREIGNIS] als Kreuzklassifikator verwendet wird, um das Merkmal VORHANDENSEIN von den anderen beiden zu trennen, und entsprechend das Schema nochmals zu vereinfachen. 


\section{Schlussfolgerungen}

Die grundlegende Idee hinter dem „Annotation Mining “ ist, eine möglichst hohe Zahl poteñiell relevanter linguistischer Eigenschaften bei einer möglichst hohen Zahl einzelner Daten zu repräsentieren und aus den Daten durch Klassifikationsverfahren diejenigen Eigenschaften zu extrahieren, die für die syntaktische Distribution etwa der PNKen relevant sein können. Anhand der Präpositionen obne und unter haben wir zeigen können, welche Merkmale die Weglassbarkeit des Artikels bedingen. Für die Präposition obne konnten wir zunächst ableiten, dass bestimmte Interpretationen der Präposition für die Weglassbarkeit des Artikels beim Komplement sprechen, andere hingegen dagegen. Eine genauere Betrachtung zeigte dann aber bereits, dass strukturelle Faktoren (was wird modifiziert) hier eine Rolle spielen könnten. Strukturelle Faktoren müssen neben den interpretatorischen Faktoren ohnehin angesetzt werden, um zu erklären, welchen Einfluss die Realisation eines postnominalen Komplements auf die Weglassbarkeit des Artikels hat.

Für die Präposition unter konnten wir diesen letzten Aspekt erneut sichtbar machen, so dass man von einer allgemeinen strukturellen Eigenschaft sprechen kann: Besitzt ein Substantiv ein postnominales Komplement, dann wird die Weglassbarkeit des Artikels erschwert, wobei dies insbesondere gilt, wenn das Komplement ein Satz ist.

Besonders auffällig ist im Vergleich der Präpositionen, dass bei obne eine Kernbedeutung den Artikelwegfall erleichtert, eine andere hingegen den Wegfall erschwert, während es bei unter nur Interpretationsmerkmale gibt, die den Artikelwegfall erschweren. Vor einer Betrachtung weiterer Präpositionen ist es sicherlich zu früh, diese Beobachtung zu werten. Dennoch bietet sich hier eine Spekulation an: Bei obne stehen die Kernbedeutungen somit für bzw. wider den Artikelwegfall (möglicherweise auch in Verbindung mit weiteren strukturellen oder interpretativen Bedingungen), bei unter ist aber ähnliches (noch) nicht zu beobachten. Ist dies vielleicht der Grund dafür, dass der Artikelwegfall bei ohne schon der Regelfall ist, bei unter aber noch die Ausnahme?

\section{Literatur}

Allan, Keith (1980): Nouns and countability. In: Language 56, 3, S. 541-567.

Baayen, R. Harald (2001): Word frequency distributions. (= Text, Speech and Language Technology 18). Dordrecht u.a.

Baayen, R. Harald (2008): Analyzing linguistic data. A practical introduction to statistics using R. Cambridge u.a.

Baldwin, Timothy et al. (2006): In search of a systematic treatment of determinerless PPs. In: Saint-Dizier, Patrick $(\mathrm{Hg})$ ): Syntax and semantics of prepositions. (= Text, Speech and Language Technology 29). Dordrecht, S. 163-179. 
Bale, Alan/Barner, David (2009): The interpretation of functional heads: using comparatives to explore the mass/count distinction. In: Journal of Semantics 26, S. $217-252$.

Barner, David/Snedeker, Jesse (2005): Quantity judgements and individuation: evidence that mass nouns count. In: Cognition 97, S. 41-66.

Borer, Hagit (2005): Structuring sense. Bd. I: In name only. Oxford u.a.

Chiarcos, Christian et al. (2008): A flexible framework for integrating annotations from different tools and tagsets. In: Traitement Automatique des Langues. Special Issue Platforms for Natural Language Processing (ATALA), 49, 2, S. 271-291.

Dömges, Florian et al. (2007): Measuring the productivity of determinerless PPs. In: Costello, Fintan/Kelleher, John/Volk, Martin (Hg): Proceedings of the 4th ACLSIGSEM Workshop on Prepositions. Prag, S. 31-37. Internet: www.linguistics.ruhr-uni-bochum. de/ kiss/publications/DKMR_3.pdf (Stand: 13.01.2011).

Duden (2002): Duden. Standardwörterbuch Deutsch als Fremdsprache. Hrsg. v. Wissenschaftlichen Rat der Dudenredaktion. Mannheim u.a.

Duden (2005): Duden. Bd. 4: Die Grammatik. Hrsg. v. Wissenschaftichen Rat der Dudenredaktion. 7., völlig neu erarb. u. erw. Aufl. Mannheim u.a.

Durell, Martin/Brée, David (1993): German temporal prepositions from an English perspective. In: Zelinsky-Wibbelt, Cornelia $(\mathrm{Hg}$ ): The semantics of prepositions. From mental processing to natural language processing. (= Natural Language Processing 3). Berlin/New York, S. 295-325.

Eberle, Kurt (2009): -ung nominalizations of verbs of saying in German: events and propositions. In: Proceedings of CHRONOS 9 - 9th International Conference on Tense, Aspect and Modality. Paris.

Espinal, M. Teresa/McNally, Louise (ersch. 2011): Bare singular nominals and incorporating verbs in Spanish and Catalan. In: Journal of Linguistics.

Evert, Stefan (2004): A simple LNRE model for random character sequences. In: Le poids des mots. Actes des 7es Journées Internationales d'Analyse Statistique des Données Textuelles. Louvain-la-Neuve, S. 411-422.

Gillon, Brendan S. (1999): The lexical semantics of English count and mass nouns. In: Viegas, Evelyne (Hg.): Breadth and depth of semantic lexicons. (= Text, Speech, and Language Technology 10). Dordrecht u.a., S. 19-37.

Harrell, Frank E. (2001): Regression modeling strategies: with applications to linear models, logistic regression, and survival analysis. New York u.a.

Hartrumpf, Sven/Helbig, Hermann/Oswald, Rainer (2003): The semantically based computer lexicon HaGenLex - structure and technological environment. In: Traitement automatique des langues 44, 2, S. 81-105.

Helbig, Gerhard/Buscha, Joachim (2001): Deutsche Grammatik. Ein Handbuch für den Ausländerunterricht. Berlin u.a.

Himmelmann, Nikolaus (1998): Regularity in irregularity: article use in adpositional phrases. In: Linguistic Typology 2, S. 315-353. 
Jespersen, Otto (1924): The philosophy of grammar. London.

Kiss, Tibor (2007): Produktivität und Idiomatizität von Präposition-Substantiv-Sequenzen. In: Zeitschrift für Sprachwissenschaft 26, 2, S. 317-345.

Kunze, Claudia/Lemnitzer, Lothar (2002): GermaNet - representation, visualization, application. In: Proceedings of the Language Resources and Evaluation Conference (LREC) 2002. Bd. V. Las Palmas de Gran Canaria, S. 1485-1491.

Le Bruyn, Bert/de Swart, Henriëtte/Zwarts, Joost (2009): Bare PPs across languages. Workshop on bare nouns. Paris.

Lezius, Wolfgang/Dipper, Stefanie/Fritschen, Arne (2000): IMSLex - representing morphological and syntactic information in a relational database. In: Heid, Ulrich et al. (Hg.): Proceedings of the 9th EURALEX International Congress. Stuttgart, S. 133-139.

Müller, Antje et al. (i.Dr.): Annotation of preposition senses in German. In: Stolterfoht, Britta/Featherston, Sam (Hg): Proceedings of Linguistic Evidence 2009. Berlin.

Müller, Christoph/Strube, Michael (2006): Multilevel annotation of linguistic data with MMAX2. In: Braun, Sabine/Kohn, Kurt/Mukherhjee, Joybrato (Hg:): Corpus technology and language pedagogy: new resources, new tools, new methods. (= English Corpus Linguistics 3). Frankfurt a.M. u.a., S. 197-214.

Schmid, Helmut (1995): Improvements in part-of-speech tagging with an application to German. In: Proceedings of the EACL SIGDAT Workshop. Dublin, S. 47-50.

Schmid, Helmut/Fitschen, Arne/Heid, Ulrich (2004): SMOR: A German computational morphology covering derivation, composition, and inflection. In: Proceedings of the Language Resources and Evaluation Conference (LREC) 2004. Lissabon, S. $1263-1266$.

Schmid, Helmut/Laws, Florian (2008): Estimation of conditional probabilities with decision trees and an application to fine-grained POS tagging. In: Proceedings of 22nd International Conference on Computational Linguistics (COLING), 18-22 August 2008, Manchester, UK. Stroudsburg, S. 465-472.

Schröder, Jochen (1986): Lexikon deutscher Präpositionen. Leipzig.

Stadtfeld, Tobias (i. Vorb.): Classifying countability. Diss. Bochum.

Stvan, Laurel S. (1998): The semantics and pragmatics of bare singular noun phrases. Diss., Evanston/Chicago. 